“C 2017 IEEE. Personal use of this material is permitted. Permission from IEEE must be obtained for all other uses, in any current or future media, including reprinting/republishing this material for advertising or promotional purposes, creating new collective works, for resale or redistribution to servers or lists, or reuse of any copyrighted component of this work in other works." 


\title{
Transdisciplinary learning in technology degrees
}

\author{
Wayne Brookes \\ Faculty of Transdisciplinary Innovation \\ University of Technology Sydney \\ Australia \\ Email: wayne.brookes@uts.edu.au
}

\begin{abstract}
This paper outlines a new kind of degree that has technology at its core, but is transdisciplinary in nature. Rather than students learning knowledge in discrete disciplinary blocks, they are exposed to ideas and practices from a wide variety of disciplines, and use these to create new ways of working that are underpinned by capabilities in computational thinking, complex systems, data and reasoning.
\end{abstract}

\section{Keywords-curriculum development;} innovation; creativity; transdisciplinary learning

technological

\section{INTRODUCTION}

Traditionally, technology degrees like engineering, computing science or information technology are specialist in nature, and in recent decades have become even more so with the rise of new technologies and new fields of practice fragmenting disciplines into niche areas. While there is no doubt that the demand for technology specialists will continue to increase, as will the number of specializations, employers are beginning to recognize a need for a new type of graduate one that can see beyond these specializations and connect ideas and practices from multiple disciplinary areas.

Universities around the world are responding in different ways to address this need for "innovative" graduates. In the simplest approach, students are encouraged to study elective courses outside their core discipline, for example to take courses in innovation and entrepreneurship. In other cases, skills such as creativity, innovation and entrepreneurship are being embedded into the core curriculum, or provided as addons or extensions. This paper outlines a more radical approach being taken at the University of Technology Sydney - the creation of an entirely new degree which is inherently transdisciplinary in nature, but underpinned by learning about technology. This paper introduces the Bachelor of Technology and Innovation, and some of its underlying design principles.

\section{TRANSDISCIPLINARITY}

Before continuing, we should consider the terms multidisciplinary, interdisciplinary and transdisciplinary. While there are numerous definitions for these terms, here we broadly align with the views expressed in [1] and [2], adapted for how they typically manifest in student project work.

In multidisciplinary learning, students stay within the boundaries of their own disciplines, but come together for the purpose of addressing a particular challenge. The different disciplines might work either in parallel to propose solutions from their discipline's perspective, or might work sequentially, where one discipline completes their component of the task, then hands over to the next discipline. While all participants are working towards the same challenge, any advances in knowledge or practice typically stay within existing discipline boundaries.

In interdisciplinary learning, there is greater collaboration between the disciplines by having students work together in mixed-discipline teams. However, while the team is working towards a common goal, each individual is still bringing their own discipline's perspective to bear on the challenge at hand. The key distinction is that students now have greater exposure to methods and practices of different disciplines, and will hopefully learn methods from other disciplines, and consider how they might apply them in their own contexts.

Transdisciplinary learning extends the idea of interdisciplinarity in that students are encouraged to move beyond their own discipline and work collaboratively to create new methods, practices and frameworks that transgress the traditional boundaries of individual disciplines. Students are operating in new, interstitial spaces between and across disciplines, and rather than just learning knowledge, they become knowledge creators in these new spaces. Rather than just transferring known methods between disciplines, transdisciplinary learning and practice involves creating new methods and practices that emerge from the intersections of disciplines.

There are a number of reasons why transdisciplinarity is important in higher education, and why now is the time. The first is the complex and interconnected nature of problems facing society today, summarized by [3] as a polycrisis - " $[. .$. a situation where there is no one, single big problem - only a series of overlapping, interconnected problems." No single discipline holds the answers to holistically addressing this notion of polycrisis - responses need to bring multiple perspectives to bear, and new practices and methods devised that blend expertise from different disciplines.

But apart from the lofty ideal of creating responses to the world's greatest problems, there is a more grounded reason why transdisciplinarity is growing in importance. The modern workforce that graduates enter already comprises multiple disciplines. A graduating IT professional entering the workforce is not going to only work with other IT staff - they may work with product designers, marketers, accountants, lawyers and more. That alone does not lead to a need for transdisciplinarity - that situation of multiple professionals 
working side-by-side has existed for many decades. However, in recent years, as professionals have become increasingly more specialized, the need for a new type of employee has begun to arise - someone who can take a broader perspective, and can help organizations move forward by generating new ideas that combine expertise from different professional areas.

A third reason is the changing nature of work, and the need for graduates to be increasingly flexible and agile throughout their career. The concept of studying a degree to prepare for one job for life is increasingly rare. Graduates are likely to move through many jobs in their careers, and possibly even different industries and types of work. And increasingly, graduates will move into new industries that didn't exist when they started their degrees [4].

The World Economic Forum report on 'The Future of Jobs' describes that we are entering what they term the fourth industrial revolution, which is going to fundamentally change the nature of work in a wide variety of industries [5]. One reason behind these changes is through advances in technology, and more importantly, where different technologies and disciplines intersect, giving rise to new opportunities. One example is 3D bioprinting, which has arisen by exploring what can happen when we combine additive manufacturing technology (3D printing) with fields including biomedical engineering, biomaterials science, cell biology and medicine [6]. While this is a sophisticated example, it demonstrates how combinations of technologies can lead to new opportunities for those with the breadth of knowledge to seek out such opportunities. It also leads to new future job opportunities - will we one day see careers for human organ designers [7]?

This paper argues that to rise to these emerging complex challenges, educational approaches that allow students to develop transdisciplinary ways of thinking and practices will create graduates who can see opportunities that span discipline boundaries, be adaptable in the changing workforce, and able to help bring about change locally in their own environment as well as responding to global challenges.

\section{Potential for Technology Education}

Transdisciplinary approaches in general should not privilege any single discipline. The methods and practices of transdisciplinary work are drawn from multiple disciplines, and depending on the project or task at hand, different bodies of knowledge and practices may take greater or lesser importance during different phases. In an education setting, the goal is to develop ways of thinking and engaging with the world through the lenses of different disciplines. In some regards, it adopts some of the ideas behind a liberal arts degree, although transdisciplinarity takes a more integrative approach.

However, the reality of today is that information technology is both a discipline in its own right, but also underpins virtually every other discipline, if not in content, then in expression and communication. Many of the changes taking place in the workforce now and over the next 10-15 years are enabled by advances in technology. Drivers of workforce and societal change include advances in computing power, device connectivity, big data, artificial intelligence, advanced robotics and the rise of peer-to-peer marketplaces (enabled by computing technology) [5, 8].

New technologies are being created at an ever-increasing pace. Companies are unsure which new technologies to adopt, and how best to incorporate them into the business and stay ahead of their competition. For society, the pace of technological change raises questions about the appropriateness of new technologies and their impacts such as privacy, politics, access and participation, sustainability, and more.

So while transdisciplinary approaches should not privilege any single discipline, it is also true that advances in computing power, connectivity, data, AI and robotics are impacting upon all disciplines. Thus to work in and across disciplines, it is vital to prepare graduates with a sound understanding of the role and future possibilities of digital technology in enabling transformation of practices, as well as the creation of new, transdisciplinary practices that arise through the application of emerging technology. It is equally, if not more, important that graduates are also able to think critically about the adoption of technology, and make decisions informed by ethical frameworks that respect the humanity of others.

Therefore this paper outlines a new type of technology degree that is transdisciplinary, but specifically develops students' technology capabilities including computational thinking (coding), systems thinking and complexity, and the ability to work with big data and engage in various kinds of reasoning about data and systems. The aim is to produce graduates who have technical competence, but are also able to work creatively, drawing ideas from different disciplines to create technologically driven and informed responses to complex challenges.

It is important to note that we are not proposing that this is a model for all technology degrees. There will always remain the need for technical specialists with deep disciplinary knowledge. The proposition here is for a new kind of technology degree that develops transdisciplinary capabilities in a technology context. This creates a new kind of graduate that is currently in short supply. It is not intended to replace the need for existing technology specializations.

There has been previous recognition of the value of multi-, inter- and transdisciplinary approaches in the context of technology education. For example, in 2002, Cox led a SIGGRAPH forum advocating the need for interdisciplinary approaches in the field of computer graphics [9]. Climate sensitive urban computing is another case where transdisciplinary practice has been applied [10]. The field of communication design, as advanced by ACM SIGDOC, also recognizes the importance of working across discipline boundaries [11].

\section{THE T-SHAPED GRADUATE}

Another way of looking at it is that we are advocating for the need to create T-shaped graduates. A T-shaped graduate is one who has depth of knowledge in at least one main discipline area, but has a working knowledge of a number of other discipline areas as well. 
The notion of preparing T-shaped graduates in technology fields is not new either. Boehm and Mobasser [12] present the case for $\mathrm{T}$-shaped software engineers, taking a systems thinking approach. Mukhtar, et al [13] advocate for the need for T-shaped professionals in the Service Science, Management and Engineering (SSME) domain.

The typical way to develop a T-shaped graduate is for students to first acquire depth of knowledge in their chosen technical specialisation (the vertical stroke of the T), and either afterwards, or alongside, they build their breadth (the horizontal stroke of the $\mathrm{T}$ ). However the transdisciplinary approach we have adopted in fact encourages students to develop a broad range of knowledge and perspectives from different disciplines first, and then later in the degree to develop their chosen specialisation area(s) - a "reverse $T$ ". Fig. 1 illustrates the difference in approach.

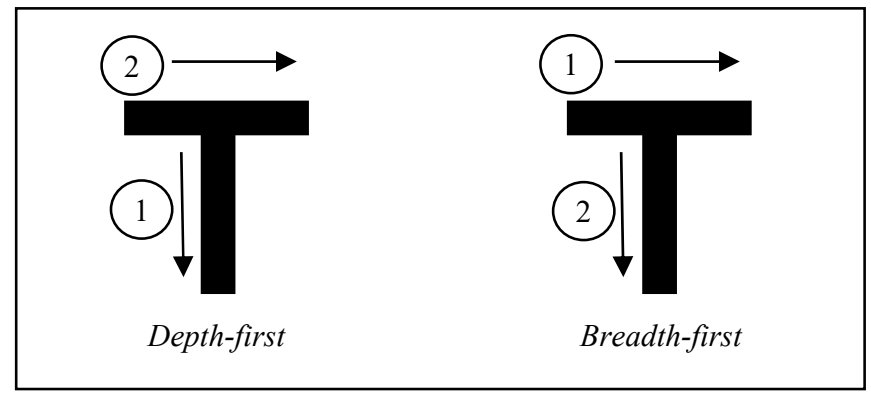

Fig. 1. Two ways of creating T-shaped graduates

In fact, beyond the T-shape, there is the possibility that students in the program may choose to have more than one specialization. This has been referred to as a pi-shaped person (where there are two specializations) or even a comb-shaped person (where there are many, but shallower areas of expertise). It is also possible that students will not develop all of these specializations during their undergraduate studies, but will extend their knowledge through postgraduate study or workplace learning.

The notion of developing some depth of expertise in multiple areas fits nicely with the notion of transdisciplinarity and the changing nature of the workforce. With the rapid pace of technological change, we already expect that university graduates will end up working in more than one field throughout their career. Having a working knowledge of a wide variety of areas, and some depth of expertise in more than one, would seem to be a good model for preparing graduates who can adapt throughout their career.

Another way of framing this discussion is to examine the role of the generalist versus the role of the specialist. These are ends of a continuous spectrum rather than discrete states, and in discussing a breadth-first or "reverse T" approach, we are not suggesting that graduates would have no specialization, although the depth of expertise would not be as great as someone completing a full three or four year specialist degree.

The role of the generalist in the professions has been raised before, for example by Costello in 2011 in the IEEE Computer
Society's IT Professional [14], where he advocates for the benefit that generalists can bring to organizations. Hardy [15] also discusses the benefits of creative generalists, and while not dismissing the need for specialists, also argues for the value that generalists bring in connecting people and ideas, and "how broad thinking leads to big ideas".

While the degree described here is not a generalist degree in the purest sense, it is also not a specialist degree, and sits somewhere on the spectrum. But even if graduates of the program do fall more on the generalist end of the spectrum, it is important to recognize the value that having such broad perspectives brings to modern organizations, which are currently oversupplied with increasingly narrow specialists.

\section{GRAduAte ATtributes}

To examine what a T-shaped, transdisciplinary, technologyfocused graduate might look like, let's examine the graduate attributes designed for the UTS Bachelor of Technology and Innovation (abbreviated BTi). There are five graduate attributes, as described below.

\section{A. Technological fluency and computational thinking}

This is the graduate attribute that enables students to develop their technical expertise. The term 'computational thinking' is used rather than simply 'coding', to emphasize that the core attribute being developed is a way of thinking about and working with algorithms and computational systems. While coding is one part of that, and every graduate will be able to code at some level, being able to navigate the computational world more broadly is perhaps more important. With the rise of coding classes in primary and high schools, within a decade, coding skills alone will not differentiate university graduates.

There will of course still be a need for specialist software engineers, as the discipline of software engineering encompasses far more than just coding. The BT $i$ degree is not intended to replace software engineering, however $\mathrm{BT} i$ graduates do need to be able to interact with professional software engineers and understand both software engineering processes as well as technical details such as algorithm design.

This graduate attribute also encompasses the ability to explore complex problems at multiple levels of abstraction, and to use technology tools to help in that process. Modelling and abstraction of systems are key concepts.

\section{B. Creating value in problem solving and inquiry}

Graduates of most degree programs these days need to demonstrate problem-solving capacity. Graduates of disciplinefocused degrees naturally adopt the problem-solving practices of that discipline. The way that engineers approach and solve problems is different to the way lawyers or designers might tackle them. Different disciplines place different value on technical correctness of solutions, stakeholder opinion of solutions, and historical precedent versus innovative approaches. 
In a transdisciplinary degree, all of these approaches are valid. Students in the BT $i$ will research and analyse problem situations from multiple disciplinary and personal perspectives.

It is also important to note that the goal is not always to solve problems. In some cases the goal is to use methods of inquiry to develop a deeper understanding of the problem space, and to frame problems in different ways that may lead to novel solutions. Or even to help organizations identify what the key problems or challenges are. Methods of inquiry from the humanities and other disciplines play a role here, yet do not often feature strongly in more traditional technology degrees.

In other cases, the problems being examined may be so large and intractable that they cannot be "solved" at all just by applying technological practices. This particularly applies in complex systems, and global problems within society, which span many areas of practice, and where there are no ideal solutions, only responses to challenges that may take steps in the right direction.

\section{Inter- and transdisciplinary practices}

While drawing in methods and practices from different disciplines is an underpinning philosophy of the entire degree, there is a graduate attribute that specifically elaborates on how graduates are expected to engage across disciplines.

A lot of the learning underpinning this attribute relates to collaboration and communication. To be an effective transdisciplinary practitioner, being able to work with others, and to elicit ideas from diverse stakeholders is a key skill.

Recognizing when collaborative approaches will lead to stronger outcomes, and understanding what is needed for successful collaboration are key attributes. But also important is the ability to assemble teams of professionals from different disciplinary backgrounds to tackle challenges. Since a graduate of a degree like the BT $i$ is not going to have the same level of technical or disciplinary expertise as a graduate of a specialist degree program, it is important that they are able to recognize the limitations of their own knowledge and when to bring in disciplinary experts to address problems and challenges, and to know which disciplinary expertise to bring for which challenges.

Being able to communicate confidently and with influence is also a key practice to work in mixed discipline teams. Graduates also need to be able to articulate complex ideas to diverse audiences, both within teams and more broadly within organizations and society.

\section{Resilient practices within complex systems}

Transdisciplinarity is well-suited to working with complexity. Complex systems are often characterized by the fact that they cross traditional discipline boundaries. Firstly, graduates must be able to abstract and represent the components of complex systems and the factors that make them complex and apply methods of inquiry to better understand the systems.

Graduates must also be able to create initiatives and responses to complex challenges. For example, graduates might generate and implement entrepreneurial or intrapreneurial initiatives, and understand the transformative nature of the work they are doing.

Overall, systems thinking is a key capability for students in this degree, as well as understanding how and when to intervene in systems to bring about positive change.

\section{E. Imaginative and ethical citizenship}

The final graduate attribute is around creative and critical thinking. As we explore the potential of technology futures, it is important that graduates consider not only the novel applications of technology (creative thinking), but also the social and ethical implications of how technology is used (critical thinking).

Students in the BTi also engage with issues of technology through the lenses of different cultural and community contexts. They need to consider the unique needs of particular communities and design culturally sensitive and appropriate technology responses.

Finally, this also involves learning about leadership - when to lead, and when to enable others to lead in order to bring technology innovation to fruition in particular organizational, community or cultural contexts.

\section{A Breadth-FIRST Degree Structure}

As previously mentioned, the approach we have taken to develop transdisciplinary capabilities in a technology context is a reverse $\mathrm{T}$ approach where the breadth comes first, and the depth of expertise later.

Fig. 2 shows the structure of the degree program, and here we describe the key components.

The degree divides into two halves. In the first three semesters, students complete subjects in three streams: technology labs, projects, and methods, practices and inquiry. In the latter three semesters, students develop their depth of expertise and build professional capabilities. This structure only describes the principal focus of each half: students will of course also begin developing professional abilities from first semester onwards, and will continue their breadth of learning in the latter part of the degree.

\section{A. Technology labs}

The technology labs are a stream of three subjects where students principally develop their technical abilities. The first subject introduces computational thinking and reasoning about data, while helping students to engage with current and emerging technologies and begin to imagine technology futures. The second subject focuses on technologies for connecting and networking people, data and ideas, and has a stronger focus on data collection, analysis and presentation. The third subject focuses on technologies for global collaboration and more advanced applications of coding and working with data. 


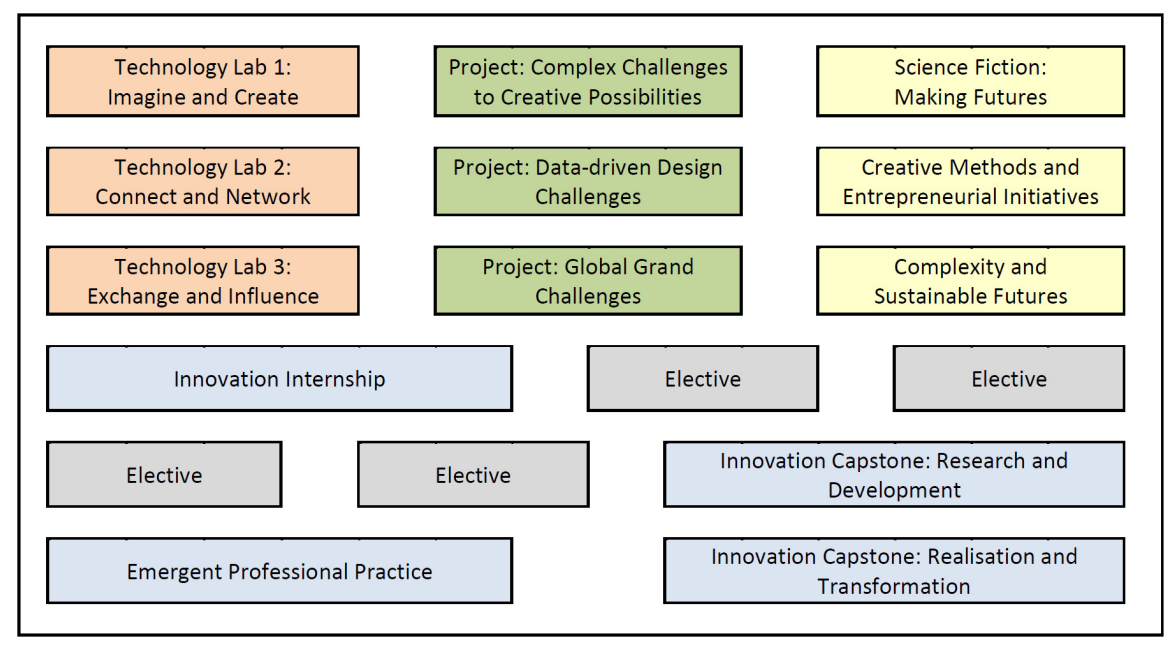

Fig. 2. Bachelor of Technology and Innovation course structure

\section{B. Complex challenge projects}

The complex challenge projects also comprise a stream of three subjects, where students work in teams on substantial and increasingly complex challenges. The first project explores problem framing and methods of inquiry rather than rushing to find solutions. The second project focuses on a data challenge, and allows students to work with multiple large data sets to identify possible initiatives to develop, and work with a client to hone their ideas, culminating in a prototype. The third project invites students to explore large-scale, complex, global challenges, and design local responses with impact.

In each of the projects, students draw on knowledge from the technology labs and methods/practices, to integrate their learning. Each project must have a technology element to it, and students also build their technical capability through experiential learning. In return, while completing the projects, students work on improving their abilities in collaboration, communication and project management, and transfer these skills into their learning in the other streams.

\section{Creative practice, methods and inquiry}

This stream of subjects helps students to develop their creative and critical thinking abilities. While creative and critical thinking is an element of the other two streams as well, it is here that students more systematically learn methods and practices taken from a wide range of disciplines, and learn how to create new methods and practices that transgress disciplinary boundaries.

Students engage more deeply with complexity (which they also encounter in the projects), and it is also in this stream where students learn about developing entrepreneurial initiatives (whether applied to the context of entrepreneurship, intrapreneurship, or social innovation). Methods for futuring, and engaging with the many dimensions of sustainability are also covered.

In this stream, as with the others, students will have academic staff from a wide range of disciplines come to the classroom at different times to help the students gain experience with ways of thinking and working in different disciplinary contexts.

\section{Professional experience and development}

The subjects in the second half of the degree are where students further develop their expertise, and begin to personalize the degree to their own interests and initial career path. They do this first through the electives, which can be chosen from a broad range of discipline areas. Students wishing to pursue more technical depth may study electives in engineering or computing. Students wishing to take a more creative path, might choose electives from design or the humanities. Students wishing to pursue an entrepreneurial future might choose electives in business and innovation.

Students also work on a substantial capstone project across two semesters, also allowing them to engage in further selflearning relating to their chosen focus or specialization.

In terms of professional development, all students complete an internship in a company that is engaged in innovation, research and development, or other forms of business transformation.

And finally, a subject to help students hone their skills in transdisciplinary professional practice. Unlike other degrees, students graduating from this program do not have a clear discipline of their own (other than a focus on technology). Therefore they need extra guidance to be able to recognize and articulate the unique value that they can bring to organizations through their transdisciplinary perspectives.

\section{CONCLUSION}

This paper outlines a new approach to technology degrees, centered around developing transdisciplinary practices. It is not intended to replace existing computer science, IT or engineering degrees - rather it is meant to produce a new kind of graduate that is not well served by existing degree structures. This new type of graduate is someone who understands how to 
work with digital technologies as a maker (not just a user), and can also use their knowledge of methods and practices from a wide range of disciplines to create new, transdisciplinary ways of working that draw on expertise from existing disciplines.

The transdisciplinary nature of the degree is evidenced in that rather than students completing a collection of existing subjects from different disciplines, instead students are engaging in experiential and inquiry-based learning in subjects where they will be exposed to academic staff from a wide range of disciplines within each subject. We have not touched much on this aspect in this paper, but it too brings challenges both for academic staff in delivering the program, as well as students in integrating learning when they are presented with so many different, and sometimes conflicting, perspectives and ways of working.

Transdisciplinary approaches generally do not privilege any specific discipline, however we argue that here it is valid to privilege technology, as it is so fundamental to society today. Computing technology and data are used in almost every field of practice, and this is only set to increase in the future. The degree described here will never develop graduates with the same depth as a computer science or software engineering degree, so the key elements we have chosen to focus on are computational thinking, and being able to work with data.

This paper has described the design of the degree, which was based on broad industry consultation, previous experiences with transdisciplinary learning in higher education, and input from stakeholders from a wide range of disciplines. Further work will include evaluation of the approaches trialed, and refinement of the program as the first cohort of students progresses through the course.

\section{ACKNOWLEDGMENT}

The design of the Bachelor of Technology and Innovation was itself a collaborative, transdisciplinary effort. I duly acknowledge the substantial contributions made to the design of this program by my many colleagues at the University of Technology Sydney involved in its creation.

\section{REFERENCES}

[1] B. Nicolescu, "The need for transdisciplinarity in higher education," Keynote speech at International Higher Education Congress, Istanbul, Turkey, May 2011.

[2] J-Y. Park and J-B. Son, "Transitioning toward transdisciplinary learning in a multidisciplinary environment," International Journal of Pedagogies and Learning, vol. 6, no. 1, 2010, pp. 82-93.

[3] S. McGregor and R. Volckmann, "Transdisciplinarity in higher education, Part 7: conclusion,” Integral Leadership Review, June 2011.

[4] Foundation for Young Australians, "The new work mindset: 7 new job clusters to help young people navigate the new work order," Report, Melbourne, Australia, 2016

[5] World Economic Forum, "The future of jobs: employment, skills and workforce strategy for the fourth industrial revolution," Report, Geneva, Switzerland, January 2016.

[6] S. Murphy and A. Atala, "3D bioprinting of tissues and organs," Nature Biotechnology, vol. 32, 2014, pp. 773-785.

[7] S. Labarre, "The most important design jobs of the future," Co.Design, April 2016.

[8] S. Hajkowicz, A. Reeson, L. Rudd, et al., "Tomorrow's digitally enabled workforce: megatrends and scenarios for jobs and employment in Australia over the coming twenty years," Report, CSIRO, Brisbane, Australia, January 2016.

[9] D. Cox, "Forum: Inspiring the renaissance person", ACM SIGGRAPH 2002 conference abstracts and applications, New York, USA, 2002, p. 17.

[10] J. Ylipulli, A. Luusua, H. Kukka and T. O. Mehlenbacher, "Winter is coming: introducing climate sensitive urban computing," Proceedings of Designing Interactive Systems, New York, USA, 2014, pp. 647-656.

[11] B. Mehlenbacher, "Multidisciplinarity and 21st century communication design," Proceedings of the ACM International Conference on Design of Communication (SIGDOC'09), New York, USA, 2009, pp. 59-66.

[12] B. Boehm and S. K. Mobasser, "System thinking: educating T-shaped software engineers," IEEE International Conference on Software Engineering, Florence, Italy, 2015, pp. 333-342.

[13] M. Mukhtar, Y. Yahya, S. Abdullah, et al, "Employability and service science: Facing the challenges via curriculum design and restructuring," International Conference on Electrical Engineering and Informatics, Selangor, Malaysia, 2009, pp. 357-361.

[14] T. Costello, "Specialists or generalists?," IT Professional, IEEE Computer Society, vol. 13, no. 6, Nov-Dec 2011, pp. 63-64

[15] S. Hardy, "The creative generalist: how broad thinking leads to big ideas," ChangeThis, Issue 19, Nov 2005. 